\title{
Investigation of Human Arm Musculoskeletal Model Using Aluminium Pole in Palm Oil Harvesting Process
}

\author{
Aliff Rahman, Shahrol Mohamaddan*, Noor Aliah \\ Abdul Majid, Magdalene Andrew Munot, Mahshuri \\ Yusuf, David Chua Sing Ngie \\ Department of Mechanical and Manufacturing, Faculty of \\ Engineering, Universiti Malaysia Sarawak (UNIMAS), \\ 94300 Kota Samarahan, Sarawak, Malaysia \\ mshahrol@unimas.my
}

\author{
Baba Md Deros \\ Department of Mechanical and Materials Engineering, \\ Faculty of Engineering \& Built Environment, Universiti \\ Kebangsaan Malaysia, 43600 UKM Bangi, Selangor, \\ Malaysia
}

\begin{abstract}
Palm oil industry is one of the important sectors in Malaysia. The growth and development in this industry shows that Malaysia is one of the largest manufactures of palm oil in the world. However this industry does causing a lot of issues in Work-related Musculoskeletal Disorders (WMSDs) especially in Harvesting Process. In addition, some of the palm oil workers in Malaysia do not take serious consideration in ergonomics awareness for their safety and health. Most of the workers are still using manual tools such as aluminium pole which has a high potential to expose to the ergonomics risk factors. Upon that situation, these studies are focusing to identify the force reaction of arm muscle and the muscle activity throughout the movement of the worker using aluminium pole in palm oil harvesting process. Inverse Dynamic Analysis is used to investigate the modelled simulation.
\end{abstract}

Keywords—Work-related Musculoskeletal Disorders (WMSDs)

\section{INTRODUCTION}

Malaysia is the second largest palm oil producer in the world which contributes to $11 \%$ from the world oil and fat production, and $27 \%$ from world export of oil and fat [1]. This statistics shows that palm oil sector give high impact to the country economy. However, there are many Work-Related Musculoskeletal Disorders (WMSDs) issues among workers' in the oil palm plantation sector. Agriculture sector is stated as one of the most dangerous industries due to many injuries and illnesses happen, such as; noise-induced hearing loss, musculoskeletal disorders, respiratory disease, increased reporting of cancer and pesticide-related illness [2].

In palm oil industry, fresh fruit bunches (FFBs) harvesting process consumes a lot of time and dealing with heavy loads, and extreme weather conditions. Awkward posture, high load and high repetition while performing the FFBs harvesting task are exposed to the risk of experiencing musculoskeletal disorders. Furthermore, in palm oil FFBs harvesting process the workers are still using manual cutting and handling tools when they are harvesting the FFBs [3].

For example, when the workers are using aluminium pole to cut the FFBs, it involves handling, lifting, placing, pull and push force, carrying and moving heavy loads [4]. These tasks might cause the worker to having forceful exertions that will place higher loads on the muscles, tendons and arm joints. These tasks may contribute to higher body demands such as higher muscle exertion as the weight of a bunch of palm oil is around 30 kilogram. Factors such as weight and height that the load has to be lifted and the lifting frequency should also be considered because they may cause high negative impact to the human body.

In addition when the work elements are performed over prolonged periods of time and repeatedly, the worker's arm are exposed to awkward postures and ergonomic risk factors. For example work element that requires frequent repetitions may lead to physical stress on the arm. It also can cause the arm to facing muscle fatigue. Muscle fatigue is known as an exercise-induced decrease in the maximal force capacity of the muscle [5]. Muscle fatigue is the decrease in ability of a muscle to generate reaction force. It may due to the vigorous exercise of work element. This situation can be extremely risky if the task requires higher focus and time consuming. Arm is one of the most affected parts that undergo musculoskeletal disorder.

Upon that situation, this study is focus to identify the force reaction of arm muscle and the muscle activity throughout the movement of the worker using aluminium pole in palm oil FFBs harvesting process. Inverse Dynamic Analysis was used to investigate the modelled simulation. It is a technique to figure out the strengths and/or moments of power (torques) by taking into account the kinematics (movement) of a body and the body's inertial properties (mass and moment of inactivity). In this research, the Anybody Modelling System ${ }^{\mathrm{TM}}$ programming is utilized [6]. The AnyBody software is an intense device that can be immeasurably utilized by examination of human foundations to recreate the musculoskeletal system. It empowers analysts to assemble and investigate a musculoskeletal model, or to make adjustment of existing models. 